\title{
lon-exchange chromatography purification of extracellular vesicles
}

\author{
Maja Kosanović, Bojana Milutinović, Sanja Goč, Ninoslav Mitić, and Miroslava Janković \\ Institute for the Application of Nuclear Energy, INEP, University of Belgrade, Belgrade, Serbia
}

BioTechniques 63:65-71 (August 2017) doi 10.2144/000114575

Keywords: extracellular vesicles; isolation; purification; amniotic fluid; ion-exchange chromatography; sucrose density gradient

Despite numerous studies, isolating pure preparations of extracellular vesicles (EVs) has proven challenging. Here, we compared ion-exchange chromatography (IEC) to the widely used sucrose density gradient (SDG) centrifugation method for the purification of EVs. EVs in bulk were isolated from pooled normal human amniotic fluid (AF) by differential centrifugation followed by IEC or sucrose density gradient separation. The purity of the isolated EVs was evaluated by electrophoresis and lectin blotting/immuno blotting to monitor the distribution of total proteins, different EVs markers, and selected N-glycans. Our data showed efficient separation of negatively charged EVs from other differently charged molecules, while comparative profiling of EVs using SDG centrifugation confirmed anion-exchange chromatography is advantageous for EV purification. Finally, although this IEC-based method was validated using $A F$, the approach should be readily applicable to isolation of EVs from other sources as well.

Most cells release extracellular vesicles (EVs) from the endosomal system (exosomes) or from plasma membranes (microvesicles) into the extracellular space (1-3). In recent years, much attention has been focused on EVs due to their pivotal role in intercellular communication and their potential as biomarkers and therapeutics (3-5). Many studies have been dedicated to the development and standardization of methods for the efficient isolation and purification of EVs; however, investigators still face problems with the purity of isolated EVs $(6,7)$. Current procedures for isolation and purification are based on different physical or biochemical properties of the EVs $(6,7)$. EVs have a size range of 30-150 $\mathrm{nm}$ (exosomes) to $>200 \mathrm{~nm}$ (microvesicles), are usually spherical in shape, and have a common set of surface molecules $(8,9)$.

Differential centrifugation, the gold standard method for EV isolation, separates EVs from other compo- nents based on size and density differences (10) and is often combined with sucrose density gradient (SDG) centrifugation, which purifies EVs according to their flotation density. Size exclusion chromatography, nano-filtration, and polyethylene glycol (PEG) precipitation separate EVs according to differences in size (11-13). Affinity/immuno-capture methods are based on interactions with specific EV surface molecules (14). However, EVs isolated using these methods are usually not pure due to contamination with protein complexes or non-EV RNA.

Starting from data indicating that EVs are electrically charged entities (15), we explored the possible use of ion-exchange chromatography (IEC) for EV purification. The negative charge on EVs results from the net negative charges of their surface molecules (glycans/phospho/sulpho groups), which should bind to an ion-exchange matrix. However, protein complexes, the main co-isolated contaminants, are expected to exist in various configurations with diverse amounts of charge and to have different interactions with the ion-exchanger. This provides a basis for their charge-resolved separation from EVs.

IEC is widely used for the separation of differentially charged molecules, which are concentrated during binding to the matrix/column and then collected in a purified, concentrated form (16). We chose amniotic fluid (AF) as a model of a complex biological fluid and compared the current protocol for isolation of amniotic fluid EVs (afEVs), based on ultracentrifugation and SDG separation $(17,18)$, with our novel protocol combining ultracentrifugation and IEC. To evaluate the purity of the isolated EVs, we monitored the total protein content, known contaminants [albumin and immunoglobulin G (lgG)], and the distributions of EV markers (CD63, CD81, and CD9), as well as the patterns of selected glycans (highmannose and sialylated N-glycans).

\section{METHOD SUMMARY}

Taking advantage of the negative charge of extracellular vesicles (EVs), ion-exchange chromatography was introduced as an alternative method for their separation and purification. Experimental conditions for charge-dependent separation of EVs from protein complexes were determined for amniotic fluid by monitoring the distribution of selected glycan/protein markers. 


\section{Materials and methods}

\section{Amniotic fluid samples}

AF samples were obtained from pregnant women who had undergone routine amniocentesis at 16-18 weeks gestation. Amniocenteses were performed due to advanced maternal age or at maternal request. After removal of cells needed for routine karyotype analysis, leftover AF, which is usually discarded, was de-identified and used for this research. Our investigation involved the use of existing human specimens and therefore is not considered research on human subjects for which informed consent is required according to guidelines of "The rules of procedures of the Ethics Committee of INEP" (No. 02-832/1). The study was approved by the local Ethics Committee of INEP, No. 03-359/1, on May $13^{\text {th }}$, 2015. Only samples with a normal fetal karyotype and no pathologies were used. Specimens were obtained from women with an average age of 36.5 years. The AF samples (16 $\mathrm{mL}$ each) were pooled to average out the heterogeneity of individual samples. Three pools, each consisting of six different samples, were examined.

Isolation of extracellular vesicles (EVs) AF pools were differentially centrifuged at $300 \times g(10 \mathrm{~min}), 3000 \times g(20$ $\mathrm{min}), 17,000 \times g$ (25 min), and 100,000 $\times g(2 \mathrm{~h}),(\mathrm{Ti} 50.2$ rotor, k-factor = 157.7) (Optima L-90K ultracentrifuge; Beckman Coulter, Indianapolis, IN). The final pellet, enriched in EVs (P100), was resuspended in $1 \mathrm{~mL} 0.05 \mathrm{M}$ Tris$\mathrm{HCl}$ buffer, $\mathrm{pH} 7.6$, by pipetting up and down 15 times and vortexing for $1 \mathrm{~min}$. Protein concentration was determined using the BCA Protein Quantification Kit (ab102536; Abcam, Cambridge, UK).

\section{Separation of amniotic fluid EVs} using a sucrose density gradient P100 preparations were separated by SDG (0.5 M, 0.7 M, 0.9 M, 1.1 M, and 1.3 M sucrose) centrifugation at 100,000 $\times g$ for 2 h (SW41Ti rotor; $\mathrm{k}$ factor = 256.6), as described earlier $(17,18)$. Twelve fractions $(1 \mathrm{~mL})$ were collected from the top of the tubes and subjected to acetone precipitation (18). The collected proteins were resuspended in an equal volume of $0.05 \mathrm{M} \mathrm{PBS}$ and used for further analysis.

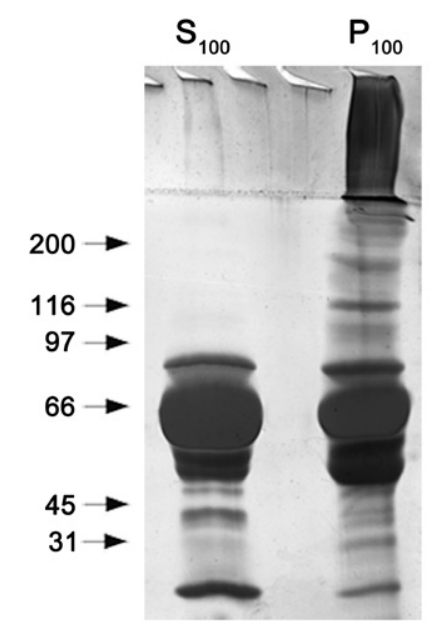

Figure 1. Protein patterns of soluble and extracellular vesicle (EV)-enriched fractions from amniotic fluid (AF). AF pools were subjected to differential centrifugation. The supernatant (S100) and pellet (P100) obtained after the final run at $100,000 \times g$ were separated by $10 \%$ SDS-PAGE (as isolated, equal volume/line), and the gel was silver stained. Arrows and numbers indicate the position of molecular mass standards in $\mathrm{kDa}$.

\section{Separation of amniotic fluid EVs} by ion-exchange chromatography DEAE Sephadex A-50 (10 mL) (Pharmacia $A B$, Uppsala, Sweden) was equilibrated with $0.05 \mathrm{M}$ Tris- $\mathrm{HCl}$ buffer, $\mathrm{pH}$ 7.6, and incubated with P100 (0.5 mL) for $2 \mathrm{~h}$ at room temperature (RT). The chromatography was performed using a gravityfed column (20 mL plastic syringe barrel filled with DEAE resin to create a column with a diameter of $2 \mathrm{~cm}$ and a height of $3.2 \mathrm{~cm}$ ), from which the eluate fractions were collected manually. The non-bound material was washed away with equilibration buffer, followed by step elution with $0.05 \mathrm{M}$ TRIS-HCl buffer, $\mathrm{pH} 7.6$, containing $0.05 \mathrm{M} \mathrm{NaCl}, 0.1 \mathrm{M} \mathrm{NaCl}, 0.2$ $\mathrm{M} \mathrm{NaCl}$ and $1 \mathrm{M} \mathrm{NaCl}$. Fractions (2 mL) were collected at a flow rate of $2 \mathrm{~mL} / \mathrm{min}$, and the elution was monitored by a lectin binding assay using Concanavalin A (Con A) (Vector Laboratories, Burlingame, CA), and CD63-, CD9-, and CD81-dot blots.

\section{Transmission electron microscopy}

Transmission electron microscopy (TEM) of EVs was performed as described previously (19). Images were collected using a Philips CM12 electron microscope (Philips, Eindhoven, The Netherlands).

\section{Concanavalin A binding assay}

Charge-resolved IEC fractions were immobilized on microwell plates (Thermo
Scientific Nunc, Rosklide, Denmark) in $0.05 \mathrm{M}$ carbonate buffer $\mathrm{pH} 9.5$ (50 $\mu \mathrm{l} /$ well) and incubated for $18 \mathrm{~h}$ at $4^{\circ} \mathrm{C}$. The Con $\mathrm{A}$ binding assay was then performed as described earlier (19).

\section{Dot blot of EV markers}

A nitrocellulose membrane (Thermo Scientific, Rockford, IL) was dotted with IEC fractions $(2 \mu \mathrm{l})$ and incubated with the following: (i) blocking solution containing 3\% BSA for $2 \mathrm{~h}$ at RT; (ii) mouse monoclonal antibody to a corresponding EV marker: anti-CD63 antibody, clone TS63 (ab59479; Abcam; RRID:AB_940915) (5 $\mu \mathrm{g} / \mathrm{mL})$ for $18 \mathrm{~h}$ at $4^{\circ} \mathrm{C}$; anti-CD9 antibody, clone CGS12A (EX201-100; Cell Guidance Systems, Cambridge, UK) (1 $\mu \mathrm{g} / \mathrm{mL})$ for 18 h at $4^{\circ} \mathrm{C}$; anti-CD81 Ab, clone CGS36K (EX203-100; Cell Guidance Systems) (1 $\mu \mathrm{g} / \mathrm{mL}$ ) for $18 \mathrm{~h}$ at $4^{\circ} \mathrm{C}$; (iii) biotinylated goat anti-mouse IgG (BA-9200; Vector Laboratories; RRID:AB_2336171) (0.75 $\mu \mathrm{g} / \mathrm{mL}$ ) for $30 \mathrm{~min}$ at RT; and (iv) avidin/ biotinylated-horseradish peroxidase (HRP) (\#PK-6100, Vector Laboratories) for 30 min at RT. Between each step, the membrane was rinsed $3 \times$ with $0.05 \mathrm{M} \mathrm{PBS}, \mathrm{pH} 7.2$ for $10 \mathrm{~min}$. Blots were visualized using Pierce ECL Western Blotting Substrate (\#32106, Thermo Scientific). TotalLab Software, version 2.00 (Amersham Biosciences, Buckinghamshire, UK) was employed for densitometry analysis.

\section{SDS-PAGE}

Samples (as isolated) were prepared in reducing Laemmli buffer and separated on a 10\% gel (20). Gels were calibrated using broad-range SDS-PAGE standards, and proteins were stained using a silver stain kit (\#161-0443, Bio-Rad, Hercules, CA).

\section{Lectin blotting and immunoblotting} Charge-resolved fractions of EVs, pooled and concentrated by ultrafiltration, or acetone-precipitated sucrose gradient fractions, were resolved on $10 \%$ gels and transferred to a nitrocellulose membrane. Membranes were blocked with 3\% BSA and subjected to lectin blotting or immunoblotting. For lectin blots, membranes were incubated with the biotinylated lectins Con A and SNA (Sambucus nigra agglutinin; Vector Laboratories) at a concentration of $1 \mu \mathrm{g} / \mathrm{mL}$ for $1 \mathrm{~h}$ at RT. Bound lectins were detected by incubation with avidin/biotinylated-HRP for $30 \mathrm{~min}$ at RT. Immunoblots were performed as described elsewhere (19). Human 
IgG was detected using biotinylated goat anti-human IgG heavy chain $(\mathrm{H})$ antibodies (BA-3080; Vector Laboratories; RRID:AB_2336154) (2 $\mu \mathrm{g} / \mathrm{mL})$ and avidin/biotinylated-HRP. Albumin was detected using rabbit anti-human albumin (Calbiochem, San Diego, CA) (1:5000) and HRP-labeled sheep anti-rabbit antibodies (AbD Serotec, Kidlington, UK) $(0.1 \mu \mathrm{g} / \mathrm{mL})$. Pierce ECL Western blotting substrate was used for visualization.

\section{Results and discussion}

As an initial step in protocols for isolation of $\mathrm{EV}$ s, differential centrifugation is known to yield concentrated EVs in the $100,000 \times$ $g$ pellet, together with co-isolated contaminants (21). When applied to AF (Figure 1), soluble proteins (S100; 100,000 $\times g$ supernatant) and those from the matching pellet (P100; 100,000 × g pellet) exhibited partially overlapping patterns in the lower molecular mass range (<80 kDa), where albumin (66 kDa) and IgG (seen as $55 \mathrm{kDa}$ heavy chain and $25 \mathrm{kDa}$ light chain) are expected to migrate as known contaminants/associated proteins (http:// microvesicles.org/gene_summary?gene_ $\mathrm{id}=213$ ) (21-23). In contrast, high molecular mass components, which could originate from different types of protein complexes or aggregates, or from afEVs themselves, were enriched in the P100 pellet.

Bulk afEVs (P100) separated by centrifugation in an SDG, which is commonly used for the removal of unwanted co-isolated proteins, and visualized in an SDS-PAGE gel are shown in Figure 2. The electrophoretic patterns of proteins in the lower sucrose density fractions (Fractions 2-5) were similar to that of the top of the gradient (Fraction 1), where soluble proteins are supposed to remain (Figure 2A). In the higher sucrose density fractions, slight differences in the removal of particular proteins of lower molecular mass (<40 kDa) were noticeable. Specifically, albumin was found to decrease across those fractions, as shown by the albumin-immunoreactive band intensity (Figure 2B), and the same was true for the IgG-immunoreactive bands (Figure 2C). In addition, the distribution of CD63 (Figure 2D), a common marker for EVs, indicated that afEVs were concentrated mainly in the low density sucrose gradient fractions (i.e. Fractions 2-4), but were also present in a mid-density fraction (Fraction 6).

Separation of afEVs using density gradient centrifugation has been reported $(17,24,25)$, but no data were given on the yield or the protein composition of the isolated populations, including the presence of known contaminants.

Our results using density gradient centrifugation showed considerable overlap of the major afEV-containing fractions with the top gradient fraction with respect to the total protein/albumin/ IgG distribution. This was in agreement with previously reported data (26), but it has not yet been resolved whether this is a general phenomenon due to the nature of EVs themselves or if it is related to the limitations of current purification methods.

Pure preparations of isolated EVs are necessary to determine whether an observed property/biological activity is intrinsic to the EVs. This is of particular importance for proper clinical and therapeutical applications in terms of exploring their multifunctional potential or diminishing the side effects of co-purified components. Given the importance of purity for downstream analysis, we examined IEC as an alternative purification step to sucrose gradient centrifugation. As negatively charged entities in a mixture of differently charged molecules, afEVs were resolved on an anion-exchange column into five fractions (Figure 3). Con A lectin, previously shown to interact strongly with EVs $(19,27)$, was used to monitor elution

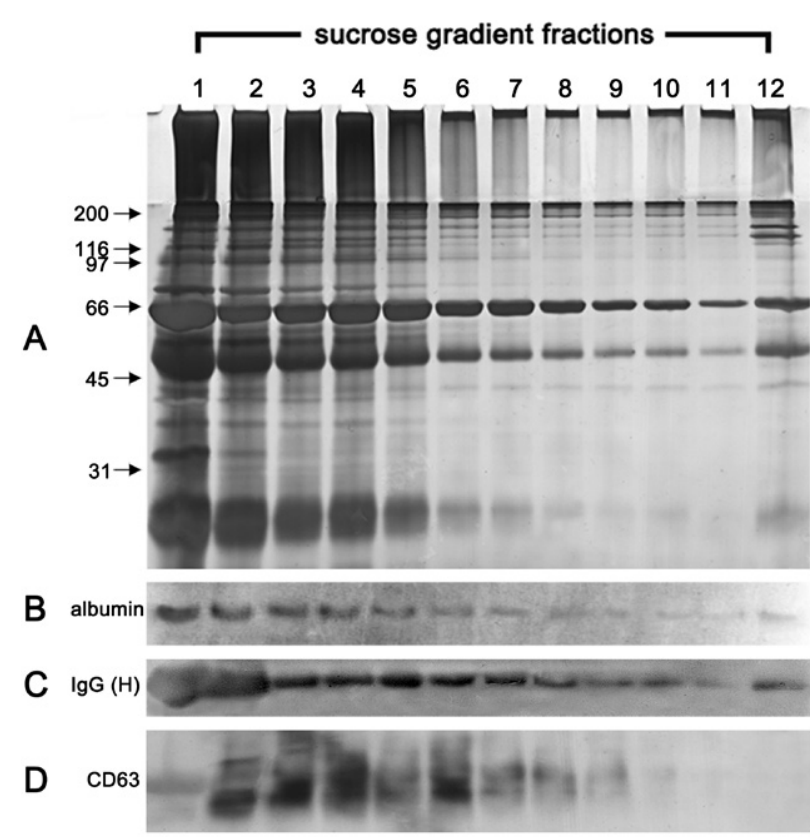

(Figure 3A). Fractions eluted with $0.2 \mathrm{M}$ $\mathrm{NaCl}$ and $1 \mathrm{M} \mathrm{NaCl}$ were strongly Con A-reactive, while weaker reactivity was observed for the non-bound fraction and the fractions eluted with $0.05 \mathrm{M} \mathrm{NaCl}$ and $0.1 \mathrm{M} \mathrm{NaCl}$.

The EV marker proteins CD63 and CD81 were found in the fractions eluted with $0.2 \mathrm{M} \mathrm{NaCl}$, and they overlapped also in the leading fractions eluted with $1 \mathrm{M} \mathrm{NaCl}$ (Figure 3B). In addition, low but detectable immunoreactivity to CD9 was observed in both of these chargeresolved populations. The presence of EVs was additionally confirmed by TEM (Figure 3C). Thus, fractions eluted with 0.2 $\mathrm{M} \mathrm{NaCl}$ contained smaller EVs, ranging 60-100 nm (Figure 3C, Panel 1), whereas in the fraction eluted by $1 \mathrm{M} \mathrm{NaCl}$, they were larger, ranging 90-220 nm (Figure 3C, Panel 2e). This indicated that in spite of the use of high salt concentrations, the separated afEVs maintained their biochemical and physical characteristics, as previously observed with human urinary EVs and mouse fibroblast $L$ cell EVs under comparable conditions $(19,28)$.

IEC separates and concentrates different components of heterogeneous samples into distinct fractions. In contrast, some currently used methods lead to sample dilution, requiring consecutive concentration steps or removal of separation media such as sucrose, all of which causes loss of EVs. Generally, it has been reported that the yield of EVs can vary considerably, from $20 \%-60 \%$ for size
Figure 2. Separation of amniotic fluid extracelIular vesicles (EVs) in a sucrose density gradient (SDG). EVs in bulk $(100,000 \times g$ pellet $)$ were separated on an SGD. Acetone-precipitated proteins from 12 gradient fractions $(1 \mathrm{~mL}$ each) were dissolved in an equal volume of PBS, separated by $10 \%$ SDSPAGE (equal volume/ line), and silver stained (A) or blotted and probed with anti-human albumin (B), anti-human immunoglobulin G (IgG) heavy chain $(\mathrm{H})$ band at $55 \mathrm{kDa}(\mathrm{C})$, or antiCD63 (D). Arrows and numbers indicate the position of molecular mass standards in $\mathrm{kDa}$. 
Figure 3. Separation of amniotic fluid extracellular vesicles (afEVs) using ion-exchange chromatography (IEC). afEVs in bulk $(100,000 \times g$ pellet $)$ were subjected to IEC on a Sephadex DEAE A-50 column eluted with $50 \mathrm{mM}$ Tris- $\mathrm{HCl}$ buffer, $\mathrm{pH} 7.6$ (a) containing increasing salt concentrations: $50 \mathrm{mM} \mathrm{NaCl}(b)$, $100 \mathrm{mM} \mathrm{NaCl}$ (c), $200 \mathrm{mM} \mathrm{NaCl}$ (d), and $1 \mathrm{M} \mathrm{NaCl}$ (e). Fractions of $2 \mathrm{~mL}$ were collected. (A) Elution of charge-resolved afEV fractions was monitored by measuring binding of solid phaseimmobilized fractions to Con A (concanavalin A) lectin. OD: optical density. (B) Elution of afEVs was monitored by measuring the reactivity of dot blot-immobilized fractions with anti-CD63, antiCD9, and anti-CD81 antibodies. A representative profile obtained by densitometry of dot signal intensities is shown. ADU: arbitrary densitometry units. (C). Transmission electron micrographs of fractions eluted with $200 \mathrm{mM} \mathrm{NaCl}$ (1) and $1 \mathrm{M} \mathrm{NaCl}$ (2). (D) Selected samples, as indicated by points in (B), from tion were subjected to $10 \%$ SDSPAGE and silver stained. Arrows and numbers indicate the position of molecular mass standards in $\mathrm{kDa}$. The arrowhead indicates the position of albumin. each charge-resolved afEV frac-
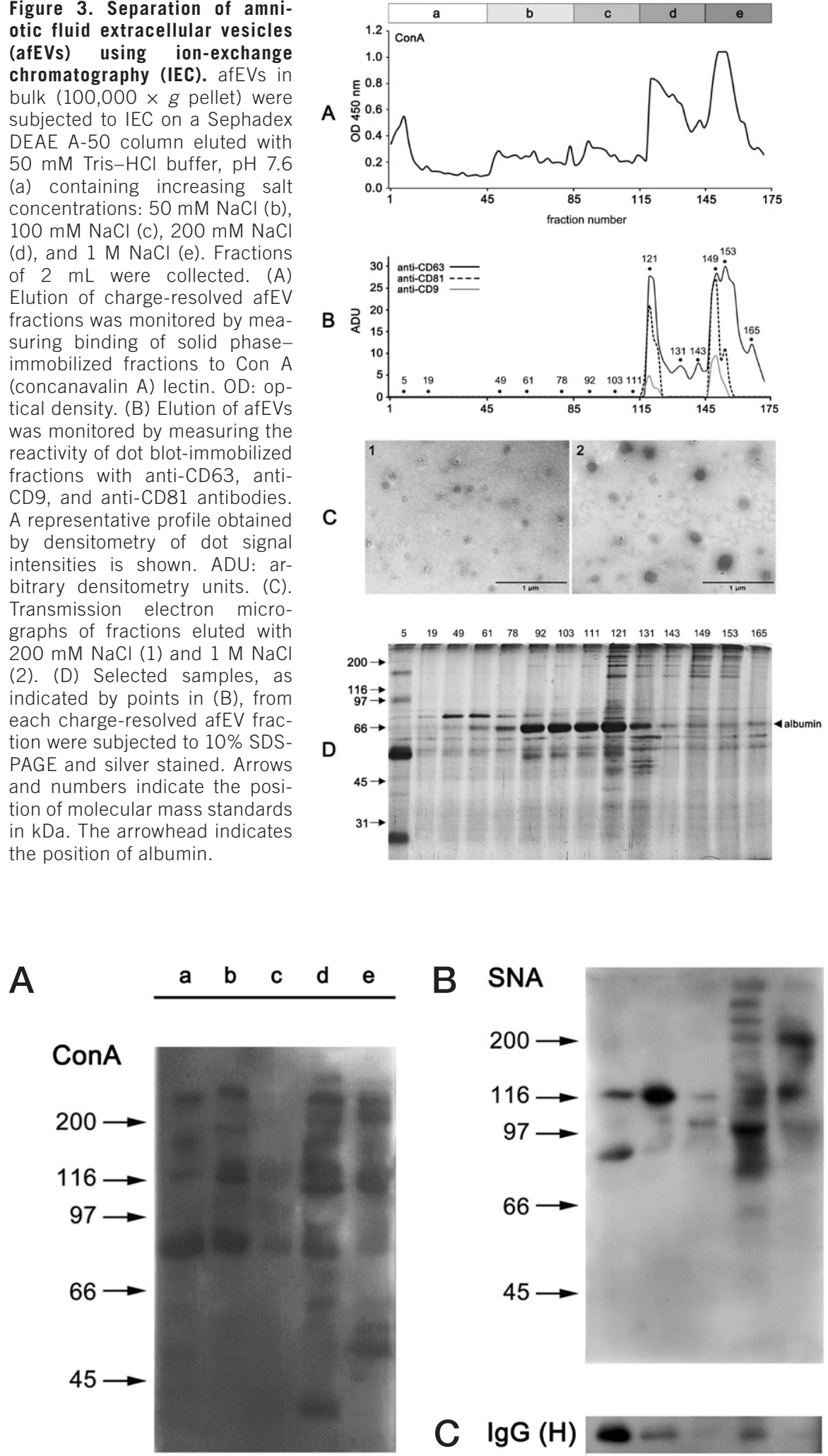

Figure 4. Glycan patterns of charge-resolved amniotic fluid extracellular vesicles (afEVs). Charge-resolved fractions of afEVs were pooled, concentrated, and subjected to $10 \%$ SDS-PAGE following lectin blotting or immunoblotting. (A) Representative patterns of bands reactive with concanavalin A (Con A) lectin. (B) Representative patterns of bands reactive with SNA (Sambucus nigra agglutinin). (C) Representative pattern of bands reactive with anti-human immunoglobulin $\mathrm{G}$ (IgG) heavy chain $(\mathrm{H})$. Numbers indicate the position of molecular mass standards in $\mathrm{kDa}$. Pooled and concentrated fractions eluted with $50 \mathrm{mM}$ Tris- $\mathrm{HCl}$ buffer, $\mathrm{pH} 7.6$ (a), containing increasing salt concentrations: $50 \mathrm{mM} \mathrm{NaCl}$ (b), $100 \mathrm{mM} \mathrm{NaCl}$ (c), $200 \mathrm{mM} \mathrm{NaCl}$ (d), and $1 \mathrm{M} \mathrm{NaCl}$ (e).
SARSTEDT

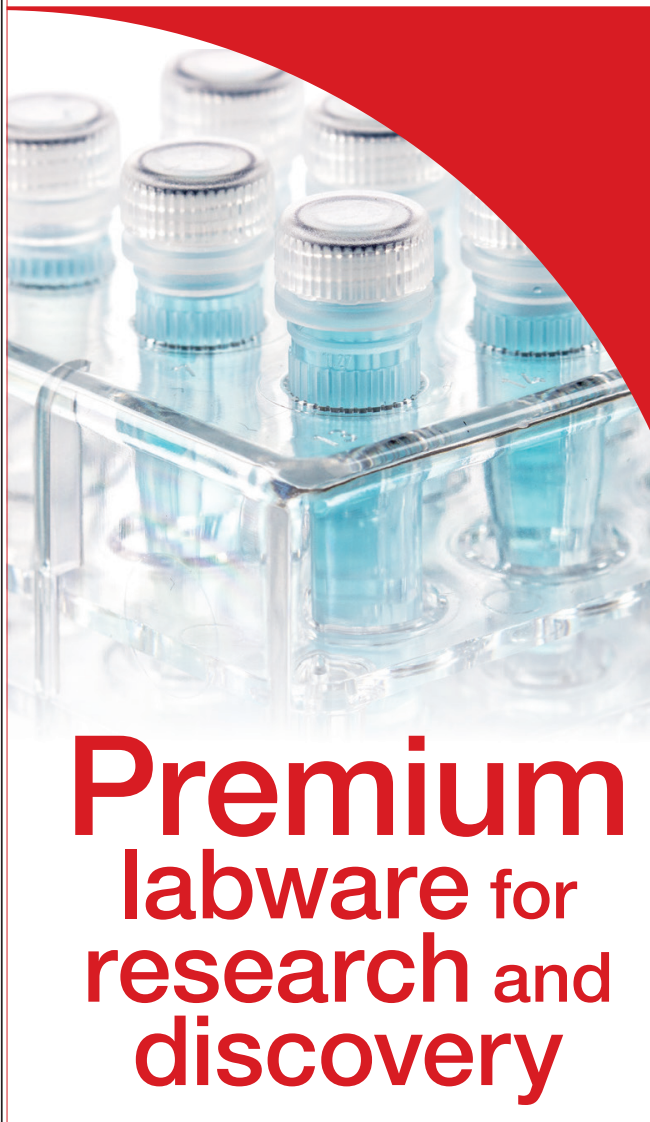

\section{Screw Cap Micro Tubes}

- Heat, freeze, store and more

- High centrifugation limits

- 3 purity standards, as well as low-binding options, for sensitive applications

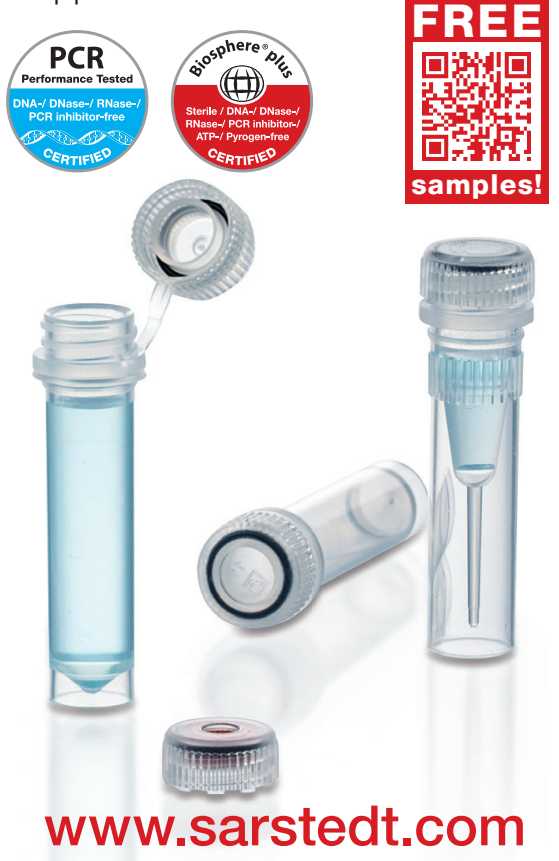


exclusion chromatography to 2\%-80\% for ultracentrifugation, and this also depends on the quality of the source material (13).

For IEC, the recovery of total protein (input) was $80 \%$. The distribution of recovered proteins was $0.6 \%$ in the non-bound fraction, $3.2 \%$ in the fraction eluted with $0.05 \mathrm{M} \mathrm{NaCl}$, and $12.5 \%$ in the fraction eluted with $0.1 \mathrm{M} \mathrm{NaCl}$. The CD63-positive fractions contained 83.8\% of the recovered protein (i.e. $55.1 \%$ in the fraction eluted with $0.2 \mathrm{M} \mathrm{NaCl}$ and $28.6 \%$ in the fraction eluted with $1 \mathrm{M} \mathrm{NaCl}$ ).

In agreement with this, the electrophoretic patterns indicated clear separation and/or enrichment of particular proteins in all five charge-resolved fractions (Figure 3D). Albumin (66 kDa band), which was used as a reference, was concentrated in the fraction eluted with $0.1 \mathrm{M} \mathrm{NaCl}$, but was less abundant in the fraction eluted with $0.2 \mathrm{M} \mathrm{NaCl}$ and was strikingly reduced in the fractions eluted with $1 \mathrm{M}$ $\mathrm{NaCl}$. SDS-PAGE of the total protein in the $0.2 \mathrm{M} \mathrm{NaCl}$ eluate revealed numerous bands in a wide molecular mass range (30 kDa-<200 kDa), resembling the total protein pattern in the low-density sucrose gradient CD63-positive fractions. The total protein pattern in fractions eluted with 1 $\mathrm{M} \mathrm{NaCl}$ was similar to that separated in mid-density SDG CD63-positive fractions.

The AF proteome is very complex, and various protein-protein or other types of interactions are possible. For example, albumin generally forms complexes with different proteins/glycoproteins, including IgG and mucins, depending on the source examined. The existence of such complexes could certainly influence the elution pattern from the ion-exchange column in terms of the distribution of particular proteins, giving wider than expected profiles based on their existing charge properties. Generally, protein complexes can be both separated as EV-associated or co-eluted with them.

Taken together, although two separated EV populations differ in charge, protein pattern, and size, it cannot be definitely confirmed at this stage that they are different with respect to type or origin.

Whereas SDG centrifugation purifies EVs according to flotation density, separation of two CD63-positive populations using IEC may also be partially related to the specificity of glycans present on their surfaces (i.e., the ligand density and steric availability of charged moieties).
The composition of charge-resolved afEVs fractions was also examined by lectin blotting (Figure 4). In contrast to the solid-phase assay, where lectin binding involves surface glycans, the patterns revealed by lectin blotting should also include cargo proteins/glycoproteins in the EVs. Each of the pooled and concentrated charge-resolved fractions of the EVs gave a complex pattern of partially overlapping bands in a wide molecular mass range when subjected to Con $A$ blotting, except for the $0.1 \mathrm{M} \mathrm{NaCl}$ eluate (Figure 4A). That fraction predominantly contains albumin, which is a non-glycosylated molecule.

Con A can bind different types of $\mathrm{N}$-glycans, including high mannose glycans (which are not sialylated) and complex and hybrid glycans (which could be sialylated). A sialic acid moiety significantly contributes to the negative charge of a molecule, so afEVs were additionally tested for SNA reactivity (Figure 4B). SNA is a sialic acid-binding lectin, recognizing Sia 2,6Gal on N-glycans and is considered to be more selective than Con A (29). Although the number of SNA-reactive bands was lower than those reacting with Con $A$, their patterns were more differentiated. There were clear differences between the SNA binding patterns of the afEV populations eluted with $0.2 \mathrm{M} \mathrm{NaCl}$ and $1 \mathrm{M} \mathrm{NaCl}$, as well as divergence in relation to the non-EVcontaining fractions.

In addition, the general patterns of $\mathrm{N}$-glycans revealed by Con A and SNA were compared with those of $\lg G$, an abundant AF glycoprotein (Figure 3C). IgG was predominantly found in the unbound fraction (56.3\%), while low lgG immunoreactivity was detected in the $0.05 \mathrm{M} \mathrm{NaCl}$ (21.6\%) and $0.2 \mathrm{M} \mathrm{NaCl}$ (14.6\%) eluates and even less in the fraction eluted with $1 \mathrm{M} \mathrm{NaCl}$ (4.8\%).

Taken together, our findings indicated simultaneous removal of major soluble contaminants as seen in the differences in the distribution of total protein/ albumin and total $\mathrm{N}$-glycans/lgG between CD63/CD81/CD9-negative and CD63/ CD81/CD9-positive fractions, as well as separation of the latter into two populations. This makes anion-exchange chromatography superior to SDG centrifugation for EV purification.

The results obtained here point to IEC as a simple and efficient method for the purification of EVs that can successfully replace SDG centrifugation. The necessity for monitoring glycans to detect fine differences in the composition of EVs was also demonstrated. Although evaluated using AF, IEC could be applied to other EV source material, given the general negative charge of EVs $(28,30)$ (Lim, S.K. 2017. Method of purifying exosomes. Patent Application. www.google.com/patents/ WO2012087241A1). However, the delineated conditions regarding the characteristics of the gradient (salt concentrations/volumes) and the choice of lectins for glycan analysis should be tested and adapted as needed. Glycoprotein composition is an important factor influencing the separation in general, and it can be tissue- and cell-specific or significantly change during growth and development. For instance, sialylation patterns in fetal tissues are different from those in adult tissues and also change during malignant transformation, depending on cancer type and progression (31).

\section{Author contributions}

M.K. and M.J. designed the study and wrote the manuscript, M.K., B.M., and M.J. interpreted the results, while M.K., B.M., S.G., and N.M. conducted the experiments.

\section{Acknowledgments}

This work was supported by the Ministry of Education, Science and Technological Development of the Republic of Serbia, project No. 173010.

\section{Competing interests}

The authors declare no competing interests.

\section{References}

1. Raposo, G. and W. Stoorvogel. 2013. Extracellular vesicles: Exosomes, microvesicles, and friends. J. Cell Biol. 200:373-383.

2. Valadi, H., K. Ekström, A. Bossios, M. Sjöstrand, J.J. Lee, and J.O. Lötvall. 2007. Exosome-mediated transfer of mRNAs and microRNAs is a novel mechanism of genetic exchange between cells. Nat. Cell Biol. 9:654659.

3. Yáñez-Mó, M., P.R. Siljander, Z. Andreu, A.B. Zavec, F.E. Borras, E.I. Buzas, K. Buzas, E. Casal, et al. 2015. Biological properties of extracellular vesicles and their physiological functions. J. Extracell. Vesicles 4:27066.

4. Simpson, R.J., J.W. Lim, R.L. Moritz, and S. Mathivanan. 2009. Exosomes: proteomic insights and diagnostic potential. Expert Rev. Proteomics 6:267-283. 
5. Fais, S., L. O'Driscoll, F.E. Borras, E. Buzas, G. Camussi, F. Cappello, J. Carvalho, A. Cordeiro da Silva et al. 2016. Evidence-Based Clinical Use of Nanoscale Extracellular Vesicles in Nanomedicine. ACS Nano 10:3886-3899.

6. Gardiner, C., D. Di Vizio, S. Sahoo, C. Théry, K.W. Witwer, M. Wauben, and A.F. Hill. 2016. Techniques used for the isolation and characterization of extracellular vesicles: results of a worldwide survey. J. Extracell. Vesicles 5:32945.

7. Witwer, K.W., E.I. Buzás, L.T. Bemis, A. Bora, C. Lässer, J. Lötvall, E.N. Nolte-'t Hoen, M.G. Piper, et al. 2013. Standardization of sample collection, isolation and analysis methods in extracellular vesicle research. J. Extracell. Vesicles 2:1-25.

8. György, B., T.G. Szabo, M. Pasztoi, Z. Pal, P. Misjak, B. Aradi, V. Laszlo, E. Pallinger, et al. 2011. Membrane vesicles, current state-of-theart: emerging role of extracellular vesicles. Cell. Mol. Life Sci. 68:2667-2688.

9. Kowal, J., G. Arras, M. Colombo, M. Jouve, J.P. Morath, B. Primdal-Bengtson, F. Dingli, D. Loew, et al. 2016. Proteomic comparison defines novel markers to characterize heterogeneous populations of extracellular vesicle subtypes. Proc. Natl. Acad. Sci. USA 113:E968-E977.

10. Raposo, G., H.W. Nijman, W. Stoorvogel, R. Liejendekker, C.V. Harding, C.J. Melief, and H.J. Geuze. 1996. B lymphocytes secrete antigen-presenting vesicles. J. Exp. Med. 183:1161-1172.

11. Alvarez, M.L., M. Khosroheidari, R. Kanchi Ravi, and J.K. DiStefano. 2012. Comparison of protein, microRNA, and mRNA yields using different methods of urinary exosome isolation for the discovery of kidney disease biomarkers. Kidney Int. 82:1024-1032.

12. Cheruvanky, A., H. Zhou, T. Pisitkun, J.B. Kopp, M.A. Knepper, P.S.T. Yuen, and R.A. Star. 2007. Rapid isolation of urinary exosomal biomarkers using a nanomembrane ultrafiltration concentrator. Am. J. Physiol. Renal Physiol. 292:F1657-F1661.

13. Böing, A.N., E. van der Pol, A.E. Grootemaat, F.A.W. Coumans, A. Sturk, and R. Nieuwland. 2014. Single-step isolation of extracellular vesicles by size-exclusion chromatography. J. Extracell. Vesicles 3:23430.

14. Clayton, A., J. Court, H. Navabi, M. Adams, M.D. Mason, J.A. Hobot, G.R. Newman, and B. Jasani. 2001. Analysis of antigen presenting cell derived exosomes, based on immuno-magnetic isolation and flow cytometry. J. Immunol. Methods 247:163-174.

15. Deregibus, M.C., F. Figliolini, S. D’Antico, P.M. Manzini, C. Pasquino, M. De Lena, C. Tetta, M.F. Brizzi, and G. Camussi. 2016. Chargebased precipitation of extracellular vesicles. Int. J. Mol. Med. 38:1359-1366.

16. Karlsson, E. and I. Hirsh. 2011. Ion Exchange Chromatography, p. 93-133. In J.-C. Janson (Ed.), Protein Purification: Principles, High Resolution Methods, and Applications. John Wiley \& Sons, Inc., Hoboken, NJ

17. Keller, S., C. Rupp, A. Stoeck, S. Runz, M. Fogel, S. Lugert, H.-D. Hager, M.S. AbdelBakky, et al. 2007. CD24 is a marker of exosomes secreted into urine and amniotic fluid. Kidney Int. 72:1095-1102.

18. Keller, S. 2009. Charakterisierung und funktionelle Analyse von Exosomen aus humanen Körperflüssigkeiten. PhD thesis. Heidelberg University.
19. Kosanovic, M. and M. Jankovic. 2014. Isolation of urinary extracellular vesicles from Tamm-Horsfall protein-depleted urine and their application in the development of a lectin-exosome-binding assay. Biotechniques 57:143-149.

20. Laemmli, U.K. 1970. Cleavage of Structural Proteins during the Assembly of the Head of Bacteriophage T4. Nature 227:680-685.

21. Webber, J. and A. Clayton. 2013. How pure are your vesicles? J. Extracell. Vesicles 2:1-6.

22. Hong, C.-S., S. Funk, L. Muller, M. Boyiadzis, and T.L. Whiteside. 2016. Isolation of biologically active and morphologically intact exosomes from plasma of patients with cancer. J. Extracell. Vesicles 5:29289.

23. Lötvall, J., A.F. Hill, F. Hochberg, E.I. Buzás, D. Di Vizio, C. Gardiner, Y.S. Gho, I.V. Kurochkin, et al. 2014. Minimal experimental requirements for definition of extracellular vesicles and their functions: A position statement from the International Society for Extracellular Vesicles. J. Extracell. Vesicles 3:26913.

24. Asea, A., C. Jean-Pierre, P. Kaur, P. Rao, I.M. Linhares, D. Skupski, and S.S. Witkin. 2008. Heat shock protein-containing exosomes in mid-trimester amniotic fluids. J. Reprod. Immunol. 79:12-17.

25. 25. Keller, S., J. Ridinger, A.-K. Rupp, J.W.G. Janssen, and P. Altevogt. 2011. Body fluid derived exosomes as a novel template for clinical diagnostics. J. Transl. Med. 9:86.

26. Musante, L., M. Saraswat, E. Duriez, B. Byrne, A. Ravidà, B. Domon, and H. Holthofer. 2012. Biochemical and physical characterisation of urinary nanovesicles following CHAPS treatment. PLoS One 7:e37279.

27. Batista, B.S., W.S. Eng, K.T. Pilobello, K.D. Hendricks-Munoz, and L.K. Mahal. 2011. Identification of a conserved glycan signature for microvesicles. J. Proteome Res. 10:4624-4633.

28. Luga, V., L. Zhang, A.M. Viloria-Petit, A.A. Ogunjimi, M.R. Inanlou, E. Chiu, M. Buchanan, A.N. Hosein, et al. 2012. Exosomes mediate stromal mobilization of autocrine Wnt-PCP signaling in breast cancer cell migration. Cell 151:1542-1556.

29. Shibuya, N., I.J. Goldstein, W.F. Broekaert, M. Nsimba-Lubaki, B. Peeters, and W.J. Peumans. 1987. The elderberry (Sambucus nigra L.) bark lectin recognizes the Neu5Ac(alpha 2-6) Gal/GalNAc sequence. J. Biol. Chem. 262:15961601.

30. Milutinovic, B., N. Mitic, J. Roncevic, S. Goc, and M. Jankovic. 2016. Glycome complexity of human seminal plasma high molecular mass components: Evaluation of the contribution of acid-soluble glycoproteins/mucins and extracellular vesicles. Arch. Biochem. Biophys. 609:20-30.

31. Varki, A. and R. Schauer. 2009. Sialic Acids, p. . In A. Varki, R.D. Cummings, J.D. Esko, H.H. Freeze, P. Stanley, C.R. Bertozzi, G.W. Hart, and M.E. Etzler (Eds.), Essentials of Glycobiology. Cold Spring Harbor Laboratory Press, Cold Spring Harbor, NY.

Received 20 March 2017; accepted 22 June 2017.

Address correspondence to Maja Kosanović, Institute for the Application of Nuclear Energy, INEP, Banatska 31b, 11080, Belgrade, Serbia. E-mail maja@inep.ac.rs

To purchase reprints of this article, contact: biotechniques@fosterprinting.com

\section{:8BioTek}

NUMBER ONE FOR:
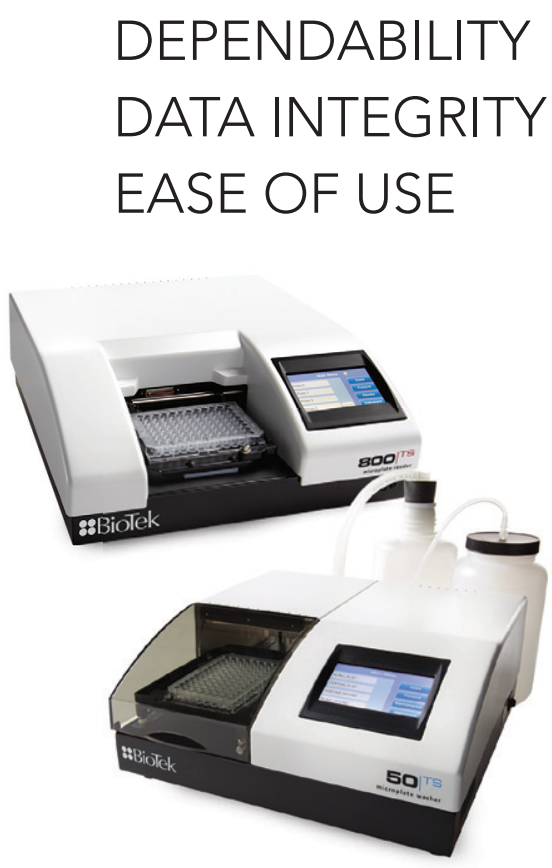

No other name is as trusted as BioTek to bring you affordability without compromising performance. www.biotek.com/readerwasher

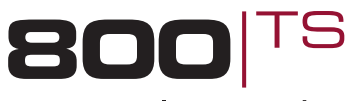

microplate reader

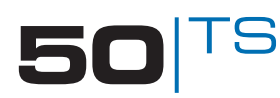

microplate washer

ISO certified and FDA registered for highest quality standards. 